\title{
Enhanced Water Resistance Performance of Castor Oil-Based Waterborne Polyurethane Modified by Methoxysilane Coupling Agents via Thiol-Ene Photo Click Reaction
}

\author{
Zhipeng $\mathrm{He}^{1,2}$, Junqi Xue ${ }^{1,2}$, Yanzi Ke ${ }^{1,2}$, Ying $\mathrm{Luo}^{1,2, *}$, Qiming $\mathrm{Lu}^{1,2}$, Yuehua $\mathrm{Xu}^{1,2}$ and \\ Chaoqun Zhang ${ }^{1,2, *}$
}

\author{
${ }^{1}$ Key Laboratory for Biobased Materials and Energy of Ministry of Education, College of Materials and Energy, South China \\ Agricultural University, Guangzhou, 510642, China \\ ${ }^{2}$ Guangdong Laboratory for Lingnan Modern Agriculture, Guangzhou, 510642, China \\ *Corresponding Authors: Chaoqun Zhang. Email: zhangcq@scau.edu.cn, nwpuzcq@gmail.com; Ying Luo. \\ Email: luoying@scauedu.cn
}

Received: 07 June 2021 Accepted: 07 July 2021

\begin{abstract}
Nowadays, waterborne polyurethanes (WPUs) prepared from renewable resources has attracted more and more attention. However, due to its structure, the prepared films easily swells in water and greatly affects the application range of WPUs. Therefore, solving the problem of water resistance is a way to improve the application range of WPUs. In this study, a series of WPU dispersions were prepared using castor oil as the bio-based polyol. Besides, the thiol-ene photo click reaction was carried out on the WPU films for silane modification. The effect of the silane modification on the chemical structures of the WPU dispersions and the properties of the WPU films was investigated and discussed. The results revealed that the WPU dispersions had a smaller particle size and potential, showing excellent stability. In addition, the modified WPU films showed highly water resistance which $72 \mathrm{~h}$ water absorption could be reduced to $1.94 \%$ and the contact angle was up to $99.34^{\circ}$. Moreover, the modified WPU films also exhibited excellent solvent resistance (in acid and salt solution) and thermal stability. This study can provide a new way to improve the water-resistance, hydrophobicity, and thermal stability of bio-based waterborne polyurethane for potential application in painting, adhesives and inks.
\end{abstract}

\section{KEYWORDS}

Castor oil; waterborne polyurethane; water resistance; silane modification

\section{Introduction}

Polyurethanes (PUs) are an important class of polymers and exhibit an exceptionally versatile range of properties and applications in coatings, adhesives, sealants, elastomers, and foams. PUs can be easily synthesized and modified ranging from high-performance elastomers to tough rigid plastics through the proper selection of reactants and processing technology [1-4]. Recently, most of the raw materials (polyols, iscoyantes, etc.) for the production of PUs are derived from fossil feedstocks. As a response to environmental concerns and increasingly depletion of fossil feedstocks, vegetable oils have been widely 
accepted as the renewable resource for the sustainable development of bio-based chemicals and polyurethane therefrom [5-9].

Among them, castor oil is one of the natural polyols that contains the hydroxyl functionality of average 2.7. Several types of PU materials and products have been developed from this cheap and readily available monomer [10,11]. Traditional solvent-based coatings contain large amounts of volatile organic compounds (VOCs), leading to public environmental and health concerns during the application and production process. Nowadays, waterborne polyurethanes (WPUs) have become one of the most attractive polymers in the past decade. WPUs using water as solvent is eco-friendly and they can not only mainly retain the properties of PUs in an organic solvent, but also exhibit the distinctive properties of low viscosity at high molecular weights, low VOCs emissions, excellent weather ability [12-14]. WPUs have become one of the most rapidly growing and active branches of polyurethane chemistry and technology. For example, Liu et al. [15] synthesized a series of castor oil based-waterborne polyurethanes through adjusting the content of 2,2-dimethylolbutanoic acid. The resulting of WPUs exhibited highly tunable properties, good biodegradability and bio-compatibility. Zhang et al. [16] obtained a series of castor oil-based waterborne polyurethanes as hair styling agent. It showed that these samples exhibited good hair-styling performance, and even better than commercial hair spray product. Zhang et al. [17] reported development of a multifunctional castor-oil-based waterborne polyurethane with high strength using controlled amounts of dithiodiphenylamine. The polymer networks possessed high tensile strength as well as excellent selfhealing efficiency. In addition, broad glass-transition temperature of the samples endowed the films with a versatile shapememory effect. Zhang et al. [18] prepared a serious of castor oil-based cationic waterborne polyurethanes and various natural phenolic acids as neutralizers. The results indicate that introduction of phenolic acids could endow polyurethane films with excellent water resistance and UV protection as well as good antibacterial performance. However, the application of WPUs are still limited by the drawback of poor water resistance of the WPU films due to large of amounts of hydrophilic groups in the polymer backbone. Therefore, water can easily enter the polymer network to swell the films, limiting its practical application [19-21].

Generally, there are three methods to improve the water resistance of WPUs coatings. (1) Decreasing the content of the ionic groups in the WPU films to reduce the hydrophilic characteristics. (2) Increasing the crosslinking densities of the polymer networks to retard the penetration of the water. (3) Introducing the low surface energy components in the WPUs network to enhance the hydrophobic characteristics.

Decreasing the contents of the carboxyl groups in the polymer backbone is a widely studied method to resolve the above problem. For example, Chen et al. [22] reported a method of reaction between 2-methyl aziridine and carboxyl group to form ester bonds, leading to the decrease of the hydrophilic groups. Tejado et al. [23] studied the acylation reaction between 1-(3-Dimethylaminopropyl)-3-ethylcarbodiimide hydrochloride and carboxyl group to generate peptide bonds, leading to the formation of 3D cross-linked networks. Rahman et al. [24] used hexamethoxymethylmelamine resin to react with carboxyl group to obtain ester bonds, resulting in the formation of cross-linked network and therefore the improvement of water resistance of the WPU films. However, the above cross-linked agents used are too expensive or toxic, which leads to the economic and environmental issue.

Introducing conjugated diene structure into WPUs followed by cross-linked reaction under UV light irradiation is also an efficient way to increase the water resistance performance of WPUs films. For example, Zhang et al. [25] introduced 2,2-bis (hydroxymethyl) butyl acrylate into the WPU backbones followed by free radical polymerization. The water absorption performance of films for $72 \mathrm{~h}$ could be reduced to $2.70 \%$ due to the highly cross-linked networks. Similarity, Kim et al. [26] used 2-hydroxyethyl acrylate (HEA) as a capping agent to introduce conjugated double bond structure in the polymer segment. The resulting WPU films exhibited a high degree of cross-linked density and low water 
absorption of $5.80 \%$ for $48 \mathrm{~h}$. Wu et al. [27] introduced dipentaerythritol hexacrylate and dipropylene glycol diacrylate into polymer chains for the production of UV-curable WPUs. It showed that the high cross-linked density of the resulting polymers demonstrated low water absorption of $3.75 \%$ for $24 \mathrm{~h}$.

In addition, reducing the surface energy and increase the hydrophobic of the polymers can also improve the water resistance. Dang et al. [28] prepared organo-silanes containing amino groups through modification of gelatin hydrolysate with KH550 and then mixed with WPU emulsions. It was found that the contact angle of the films with reduced surface energy could be high up to $90^{\circ}$ and the waterresistance of the composite films was greatly improved. Zheng et al. [29] prepared a serious of WPU films containing fluorine and silicon by using polytrifluoropropylsiloxane as a capping agent. Due to the introduction of high content of low surface energy element, the water absorption of WPU films was reduced to $5.40 \%$ for $24 \mathrm{~h}$. Wu et al. [30] prepared the aqueous hybrid silica sol using 3-Glycidyloxypropyltrimethoxysilane and tetraethylorthosilicate as the raw materials and incorporated them into WPUs through the sol-gel process. The result showed that the formation of polysiloxane crosslinked networks greatly enhanced the water resistance (water absorption low to $2.60 \%$ for 7 days). Fan et al. [31] synthesized the water-resistance WPUs by introducing flexible fluorinated siloxane units. The water absorption of the films could be reduced to $3.07 \%$ for $24 \mathrm{~h}$.

In this study, castor oil-based WPUs were synthesized with the dimethylol propionic acid (DMPA) as the internal emulsifier. Then, two kinds of methoxysilane (3-mercaptopropyltrimethoxysilane, 3mercaptopropylmethyldimethoxysilane) were used to modify the WPUs films. Centrifuge and zeta-sizer were used to characterize the stability of the WPUs. The structure and performance of the WPU films were characterized by Fourier transform infrared (FTIR), thermogravimetric analysis (TGA), differential scanning calorimetry (DSC). The effect of the silane modification on the performance of the resulting WPU films was studied and discussed.

\section{Material and Methods}

\subsection{Materials}

Castor oil (-OH number: $164 \mathrm{mg} \mathrm{KOH} / \mathrm{g}$ ) was purchased from Fuyu Chemical Co., Ltd., Isophorone diisocyanate (IPDI) was purchased from Wengjiang Chemical Reagent Co., Ltd., Dimethylol propionic acid (DMPA) and Triethylamine (TEA) were purchased from Aladdin reagent. Dibutyltin dilaurate (DBTDL) and Sodium chloride $(\mathrm{NaCl})$ was purchased from Fuchen Chemical Reagent Factory. Sodium hydroxide $(\mathrm{NaOH})$ was purchased from General reagent. 3-Mercaptopropyltrimethoxysilane (MPTS) was purchased from Shanghai Dingxian Biological Technology Co., Ltd., China. 3Mercaptopropylmethyldimethoxysilane (MPMDS) was purchased from Energy Chemical Reagent. 1173 was purchased from German Ryoji Chemical Co., Ltd., German. Methyl ethyl ketone (MEK) and Hydrochloric acid $(\mathrm{HCl})$ was purchased from Guangzhou Chemical Reagent Factory. All materials were used as received without further purification.

\subsection{Preparation of Castor Oil-Based Anionic WPU Dispersions (WPUDs)}

The preparation of anionic WPUDs dispersion from castor oil was shown in Fig. 1. Castor oil, IPDI, and DMPA were mixed into a dried double-neck flask and stirred $(220-250 \mathrm{r} / \mathrm{min})$ at $78^{\circ} \mathrm{C}$ for $10 \mathrm{~min}$ to obtain a homogenized mixture. Then several drops of DBTDL were added into the mixture as catalyst. When the mixture almost could not flow, MEK was added to reduce the viscosity of the pre-polymer. After reaction for another $2 \mathrm{~h}$, the mixture was cooled to room temperature. Next, TEA was added to neutralize free $-\mathrm{COOH}$ in the polymer chain under stirring for $30 \mathrm{~min}$. Finally, the stirring rate was added to $800 \mathrm{r} / \mathrm{min}$ that the polymer was dispersed in appropriate deionized water for $2 \mathrm{~h}$. The WPUDs were synthesized 
with DMPA and a variable ratio between the $\mathrm{OH}$ groups in castor oil, the NCO groups in IPDI, and the $\mathrm{OH}$ groups in the emulsifier $(1: 1.7: 0.69 ; 1: 2: 0.99)$ with solid content with $10 \mathrm{wt} \%$ were obtained after removal of the MEK by rotary evaporation [5].

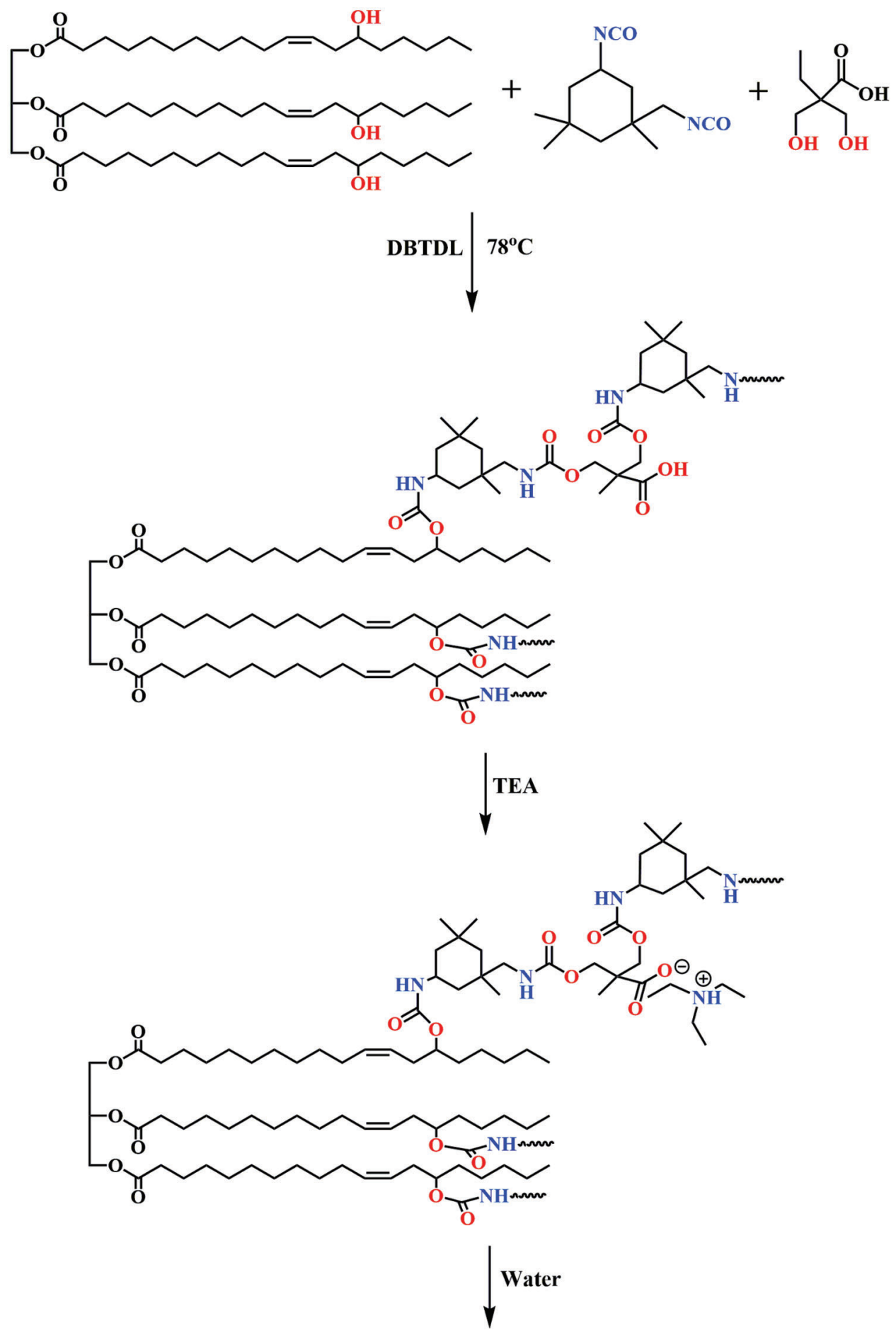

Castor oil-based anionic waterborne polyurethane dispersions

Figure 1: Synthetic route of the castor oil-based anionic WPUDs 


\subsection{Preparation of Modified Films with Mercaptosilane}

$15 \mathrm{~g}$ emulsion was poured into siliconized glass dishes and allowed the water evaporation at room temperature for 3 4 days to obtain WPU films, and the films were dried in the oven for 1 day. Then dried films were cut into predetermined size and put into quartz tubes. Next, appropriate MPTS or MPMDS was poured and several drops of 1173 were added into the tubes. Then, the thiol-ene photo click reaction was processed under UV irradiation $(350 \mathrm{~W}, 365 \mathrm{~nm})$ for $3 \mathrm{~h}$ at room temperature. After that, these samples were placed in the atmosphere at room temperature for 3 days to carry out the hydrolysis reaction and cross-linked reaction of active methoxysilane. Finally, the modified WPU films were obtained (Tab. 1 and Fig. 2).

Table 1: Compositions of the modified films

\begin{tabular}{llllll}
\hline Sample & $\begin{array}{l}\text {-OH } \\
\text { (castor oil) }\end{array}$ & $\begin{array}{l}\text { Molar ratio-NCO } \\
\text { (IPDI) }\end{array}$ & -OH (emulsifier) & MPTS & MPMDS \\
\hline $0.99-$ MPTS & 1.00 & 2.00 & 0.99 & $\sqrt{ }$ & \\
$0.69-M P T S$ & 1.00 & 1.70 & 0.69 & $\sqrt{ }$ & \\
$0.99-M P M D S$ & 1.00 & 2.00 & 0.99 & & $\sqrt{ }$ \\
$0.69-M P M D S$ & 1.00 & 1.70 & 0.69 & & $\sqrt{ }$ \\
\hline
\end{tabular}

\subsection{Characterization}

All the samples were centrifuged at $3000 \mathrm{r} / \mathrm{min}$ for $30 \mathrm{~min}$ on a Tomos 3-18 centrifuge to evaluate their stability. The zeta potential, average particle size, and particle size distributions of the samples were obtained on a zeta-sizer Nano ZSE (Malvern Instruments) at $25^{\circ} \mathrm{C}$. The samples were diluted with deionized water to about $0.01 \mathrm{wt} \%$ before a test.

FTIR spectra of the modified and non-modified films were used a Nicolet IS10 Fourier transform infrared spectrometer. Each sample was scanned at a resolution of $4 \mathrm{~cm}^{-1}$ in the range from 500 to $4,000 \mathrm{~cm}^{-1}$.

The thermo-gravimetric analysis of the modified films was carried out by a Netzsch-STA 449C thermal analyzer. All the samples $(5-10 \mathrm{mg})$ were heated under nitrogen from 30 to $700^{\circ} \mathrm{C}$ with a heating rate of $10{ }^{\circ} \mathrm{C} / \mathrm{min}$.

The differential scanning calorimetry of the modified films was carried out by a Seiko DSC 220. All the samples $(5-10 \mathrm{mg})$ were heated from -50 to $180^{\circ} \mathrm{C}$ with a heating rate of $5^{\circ} \mathrm{C} / \mathrm{min}$.

The hydrophobicity of the films was assessed by measuring the waterproof capability of modified films using a contact angle goniometer (Powereach JC2000C1). Water contact angle of the samples was measured at room temperature by the sessile-drop method.

The water-resistance of the modified films was measured by using dried films of $10 \mathrm{~mm} \times 10 \mathrm{~mm}$ (length $\times$ width) and were immersed in deionized water at room temperature for $72 \mathrm{~h}$. The weights of the modified films before and after immersion were measured. The water absorption ratios (WAR) of the modified films were calculated by the following equation:

$W A R=\frac{\mathrm{m}_{1}-m_{0}}{m_{0}} \times 100 \%$

where $\mathrm{m}_{0}, \mathrm{~m}_{1}$ represents the weights of the films before and after the immersion of water. Average values of at least three replicates of each sample were taken. 


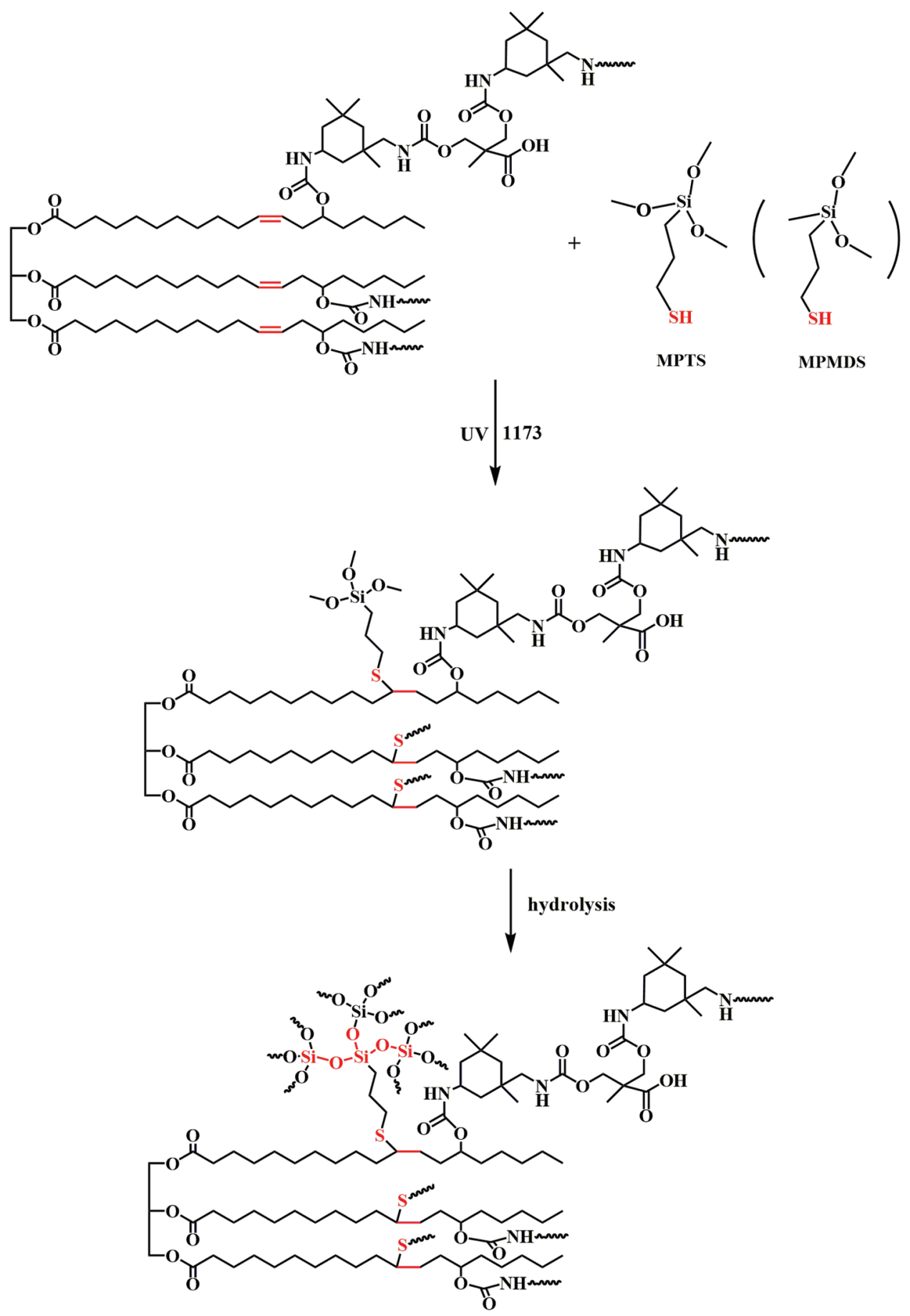

Figure 2: Synthetic route of the WPU films modified with methoxysilane

The gel content of the modified films was measured by using dried films of $10 \mathrm{~mm} \times 10 \mathrm{~mm}$ (length $\times$ width) and was immersed in THF at room temperature for $24 \mathrm{~h}$. The weights of the samples before and after immersion were measured. The gel content of the modified films was calculated by the following equation: 
Gelcontent $=\frac{\mathrm{m}_{1}}{m_{0}} \times 100 \%$

where $\mathrm{m}_{0}, \mathrm{~m}_{1}$ represents the weights of the films before and after the immersion of THF. Average values of at least three replicates of each sample were taken.

The chemical resistance of the modified films was determined by immersing them into various aqueous solutions of $1 \mathrm{~mol} / \mathrm{L} \mathrm{HCl}, 1 \mathrm{~mol} / \mathrm{L} \mathrm{NaOH}$, and saturated $\mathrm{NaCl}$ at room temperature for $24 \mathrm{~h}$. Weight change percentages of the modified films were calculated from the weight differences between the dry films and those immersed into various chemicals.

\section{Results and Discussion}

\subsection{Synthesis and Characterization of Castor Oil Based WPUDs}

The particle size distributions of the WPUDs with emulsifier hydroxyl molar ratio of 0.69 and 0.99 are presented in Fig. 3. The average particle sizes and zeta potentials data are summarized in Tab. 2. The two WPUDs have a small particle size ranging from 180-200 nm and WPUDs prepared with emulsifier hydroxyl molar ratio of 0.69 is larger than those prepared with emulsifier hydroxyl molar ratio of 0.99 (see Fig. 3). For WPUDs prepared with hydroxyl molar ratio of 0.69 , the low ionic content results in a thinner electrical double layer around the emulsion's particle, leading to the reduction of the electrostatic repulsive forces between the emulsion's particles, and therefore a larger particle size. Both the two dispersions have low negative zeta potential under $-30 \mathrm{mV}$ (see Tab. 2), exhibiting the excellent stability of the dispersions. The absolute value of zeta potential has a similar trend as the particle size. The WPUDs with emulsifier hydroxyl molar ratio of 0.99 exhibits a lower zeta potential value (absolute value) than the WPUDs with emulsifier hydroxyl molar ratio of 0.69 [5].

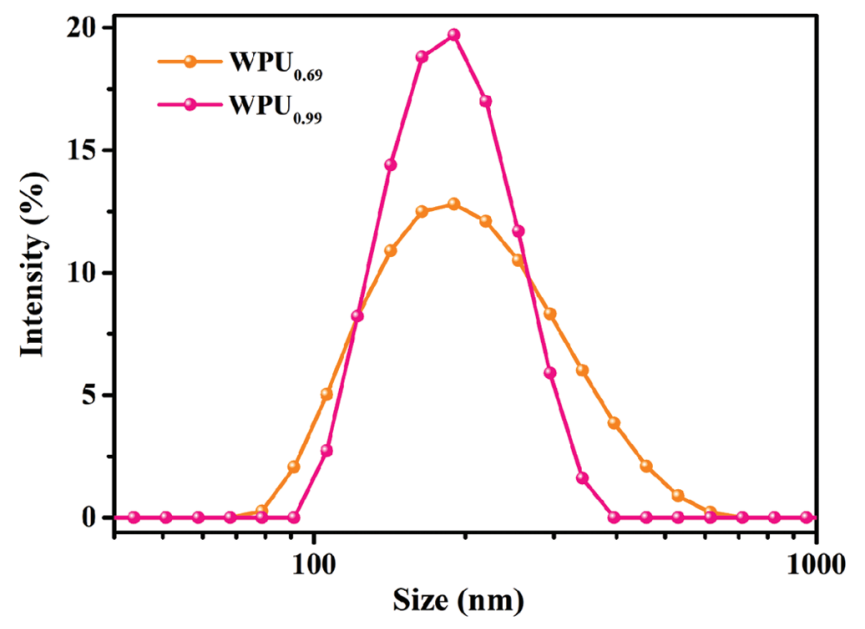

Figure 3: The particle size distribution of WPUDs with hydroxyl molar ratio of 0.99 and 0.69

Table 2: The particle size distribution and zeta potential of the WPUDs

\begin{tabular}{lllll}
\hline Sample & Appearance & Storage life & Z-average size $(\mathrm{nm})$ & Zeta potential $(\mathrm{mV})$ \\
\hline DMPA-0.69 & Milky White & $>15$ months & 209.8 & -33.8 \\
DMPA-0.99 & Milky White & $>15$ months & 187.4 & -41.0 \\
\hline
\end{tabular}




\subsection{The Methoxysilane Modification of WPU Films}

The FTIR spectra of WPU films modified with MPTS and MPMDS are displayed in Fig. 4. The peaks at $760 \mathrm{~cm}^{-1}$ is attributed to the cis-bending vibration of the carbon-carbon double bond and the peak at $3,009 \mathrm{~cm}^{-1}$ is attributed to the stretching vibration of the carbon-hydrogen single bond which only exists in the non-modified film. After modification with methoxysilane, these peaks disappear and the peak at $1,080 \mathrm{~cm}^{-1}$ and at $1,010 \mathrm{~cm}^{-1}$ which are attributed to the symmetrical stretching vibration of Si-O-C and the anti-symmetric stretching vibration of Si-O-Si appear. These results demonstrate that the thiol-ene photo click reaction of castor oil and methoxysilane was completed and the hydrolysis reaction was also completed [32].

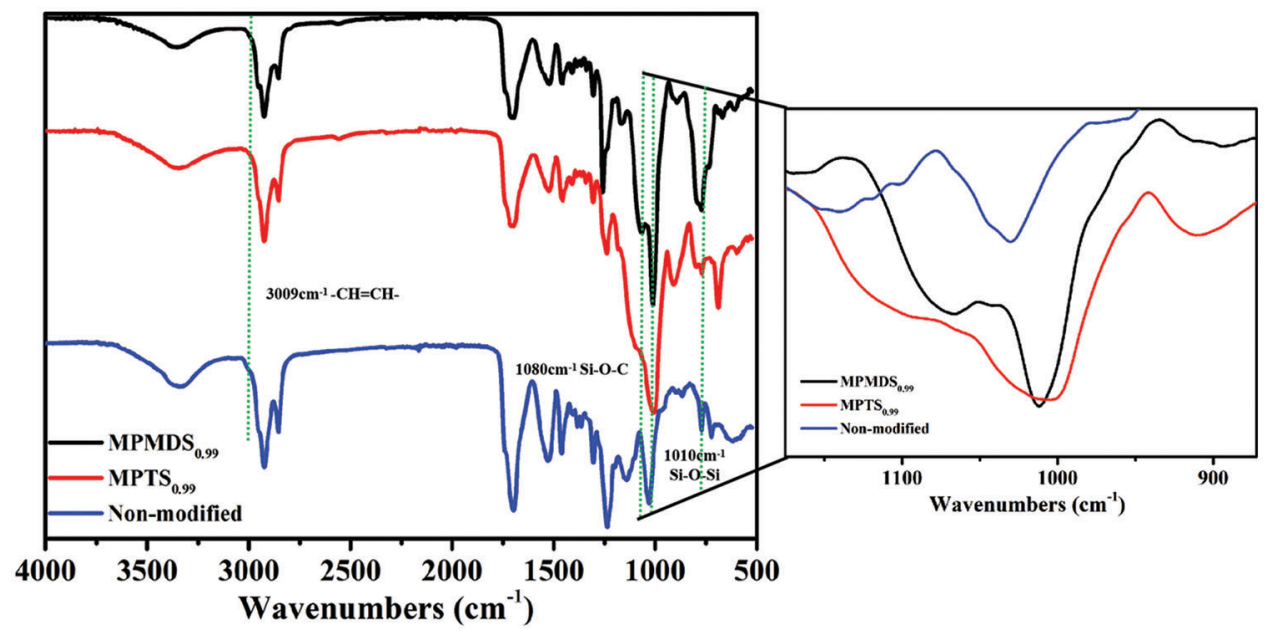

Figure 4: ATR spectra with the films of $\mathrm{MPMDS}_{0.99}, \mathrm{MPTS}_{0.69}$, and non-modified

\subsection{Properties of WPU Films Modified by Methoxysilane}

The water-resistance performance of the WPU films was evaluated with the water absorption of the films. As shown in Fig. 5 and Tab. 3, the modified WPU films show low water absorption in the range of 2.55-3.48 wt\% and the non-modified sample shows high water absorption of $18.95 \mathrm{wt} \%$. It shows that after being modified with methoxysilane, the modified films showed higher water resistance than nonmodified film due to the silicon layer covering on the surface. Besides, the WPU films modified by MPMDS have higher water absorption than WPU films modified by MPTS. The reason is that MPMDS has only two methoxy, much less than MPTS. Therefore, the poorer degree of hydrolysis is, the lower degree of cross-linked density will be. Also, the high cross-linked density leads to the dense structure on a microscopic level, resulting in the prohibition of water infiltrating into the films and enhanced water resistance performance.

In addition, the films with emulsifier hydroxyl molar ratio of 0.99 have higher water absorption than the other one. This is because the more emulsifier used, the more hydrophilic group existed and that will enhance the films hydrophilic. So, with emulsifier hydroxyl molar ratio of 0.99 , the water resistance performance of the film is poorer than the other one. In summary, among the modified films, the best water resistance film is MPTS $_{0.69}$ which water absorption is $1.94 \%$ and the worst water resistance film is MPMDS $_{0.99}$ which water absorption is $3.48 \%$. 


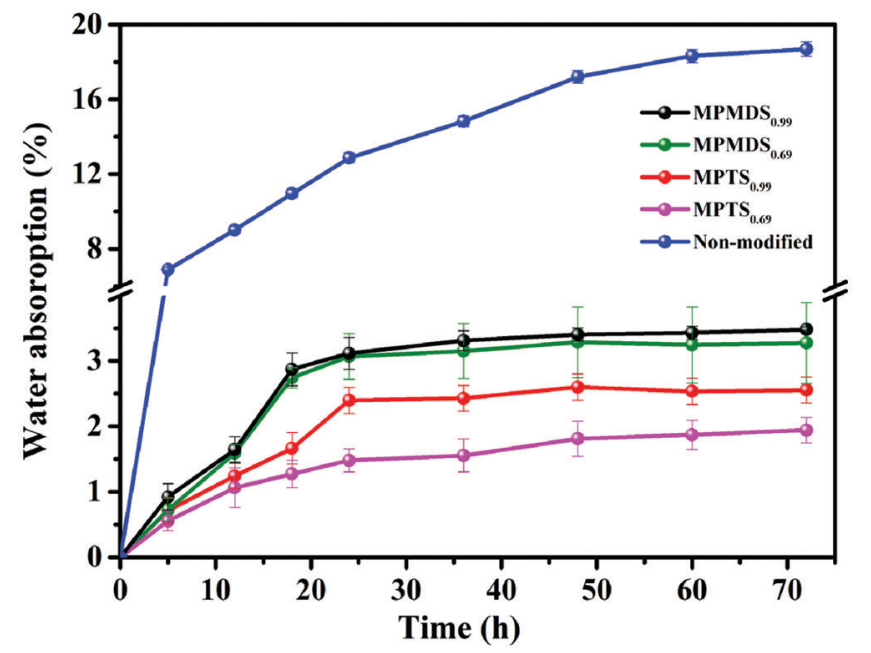

Figure 5: Water absorption for the films with different films

Table 3: The water absorption of films

\begin{tabular}{ll}
\hline Sample & Water absorption after immersion for $72 \mathrm{~h}(\%)$ \\
\hline MPTS $_{0.99}$ & $2.55 \pm 0.20$ \\
MPTS $_{0.69}$ & $1.94 \pm 0.19$ \\
MPMDS $_{0.99}$ & $3.48 \pm 0.19$ \\
MPMDS $_{0.69}$ & $3.27 \pm 0.04$ \\
Non-modified & $18.95 \pm 0.38$ \\
\hline
\end{tabular}

Gel content of the modified films plays an important role in determining the coating performance especially in water resistance and hydrophobicity. As evidenced in Fig. 6, the gel content of the MPTS $_{0.99}$, MPTS $_{0.69}$, MPMDS $_{0.99}$, MPMDS $_{0.69}$, non-modified samples are 98.94\%, 98.62\%, 85.17\%, $84.96 \%, 80.47 \%$, respectively. The gel content is greatly affected by the degree of hydrolysis of the methoxysilane. As expected, higher gel content is obtained with films modified by MPTS due to its high degree of hydrolysis. ${ }^{23}$ Chemical resistance is primarily dependent on the cross-linked density and the chemical stability of the bonds in a system. It is observed that the weight change of the modified WPU films are less than $1 \mathrm{wt} \%$ in $1 \mathrm{~mol} / \mathrm{L} \mathrm{HCl}$ and saturated $\mathrm{NaCl}$. This is due to the high degree of hydrolysis with methoxysilanes enhanced the cross-linking density of the modified WPU films which prevented the penetrating of the acid and salt solutions into the films. However, the films were fragmented in $1 \mathrm{~mol} / \mathrm{L} \mathrm{NaOH}$. This might be due to the waterborne polyurethane contains lots of carboxyl groups which was hydrolyzed in an alkaline environment. Fig. 7 shows the water contact angle images of the WPU films prepared with different emulsifier hydroxyl molar ratio of and modified with different methoxysilanes, and their data are summarized in Tab. 4. The samples of $\mathrm{MPMDS}_{0.69}$ and MPMDS $_{0.99}$ have a water contact angle below $81^{\circ}$ while the samples of MPTS 0.99 and $\mathrm{MPTS}_{0.69}$ have a water contact angle above $90^{\circ}$, exhibiting a hydrophobic surface. All the modified WPU films have lager contact angle than non-modified film. This phenomenon could be explained by the fact that the surface of the films was covered with a silicon layer that can block moisture from infiltrating the surface. In addition, the more methoxies exist, the higher degree of hydrolysis will be. 


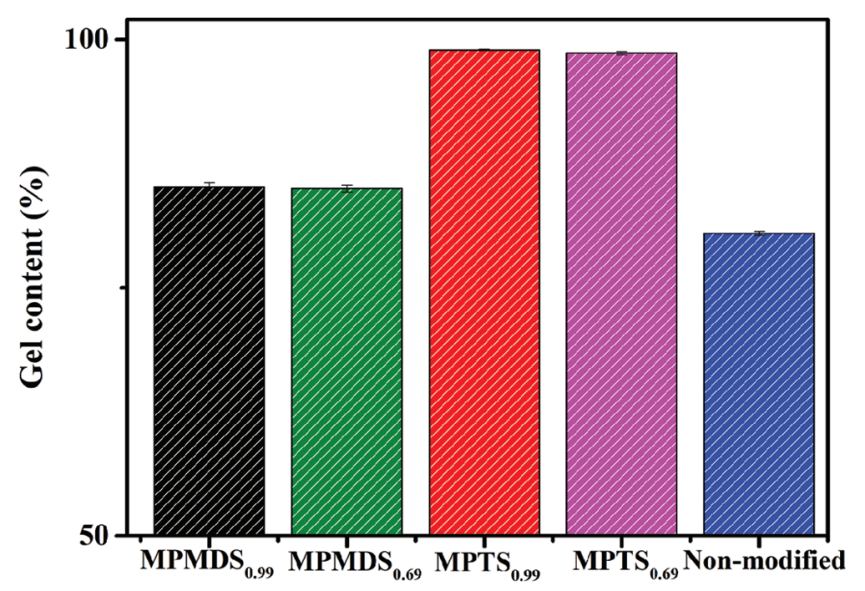

Figure 6: Gel content of the WPU films modified with different films

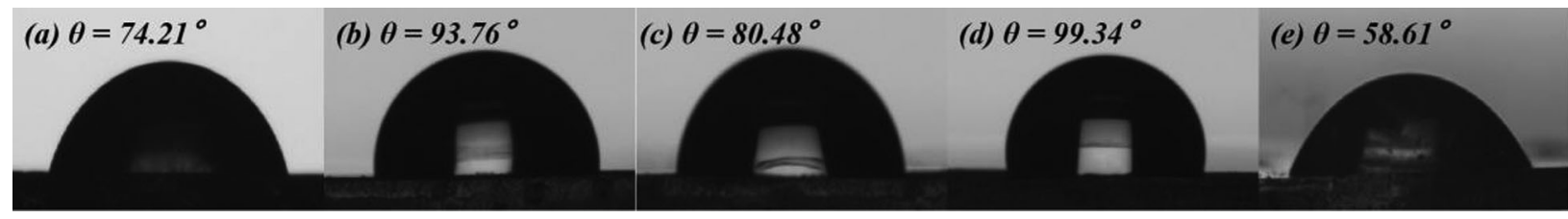

Figure 7: Water contact angle images of different films: (a) $\mathrm{MPMDS}_{0.69}$; (b) $\mathrm{MPTS}_{0.69}$; (c) $\mathrm{MPMDS}_{0.99}$; (d) MPTS $_{0.99}$; (e) Non-modified

Table 4: Chemical resistance and water contact angle of the WPU films modified with different methoxysilanes and non-modified

\begin{tabular}{lllll}
\hline \multirow{2}{*}{ Sample } & \multicolumn{3}{c}{ Weight change (\%) } & \multirow{2}{*}{\begin{tabular}{l} 
Water contact angle \\
\cline { 2 - 4 }
\end{tabular}} \\
\cline { 2 - 4 } & $1 \mathrm{~mol} / \mathrm{L} \mathrm{HCl}$ & $1 \mathrm{~mol} / \mathrm{L} \mathrm{NaOH}$ & saturated NaCl & \\
\hline MPTS $_{0.99}$ & $0.461 \pm 0.12$ & $\times$ & $0.391 \pm 0.09$ & $99.34 \pm 2.31$ \\
MPTS $_{0.69}$ & $0.260 \pm 0.14$ & $\times$ & $0.965 \pm 0.11$ & $93.76 \pm 3.15$ \\
MPMDS $_{0.99}$ & $0.870 \pm 0.22$ & $\times$ & $0.984 \pm 0.28$ & $80.48 \pm 4.37$ \\
MPMDS $_{0.69}$ & $0.693 \pm 0.18$ & $\times$ & $0.971 \pm 0.23$ & $74.21 \pm 3.58$ \\
Non-modified & $13.89 \pm 0.31$ & $\times$ & $3.27 \pm 0.21$ & $58.61 \pm 4.02$ \\
\hline
\end{tabular}

Note: $\times$ indicating the fragment of the WPU films.

TGA profiles of the resulting films are shown in Fig. 8a and the TGA data including $5 \mathrm{wt} \%$ loss temperature $\left(T_{5}\right.$, the onset decomposition temperature), $10 \mathrm{wt} \%$ loss temperature $\left(T_{10}\right), 50 \mathrm{wt} \%$ loss temperature $\left(T_{50}\right)$, and the maximum rate of weight loss temperature $\left(T_{\max }\right)$ [5] are tabulated in Tab. 5. As well known, WPU films exhibited onset decomposition temperature due to the existence of the labile urethane groups. The onset decomposition temperature of WPU films modified with MPMDS is below $220^{\circ} \mathrm{C}$ while the onset decomposition temperature of modified with MPTS is nearly $280^{\circ} \mathrm{C}$, much higher than those of the non-modified films $\left(165^{\circ} \mathrm{C}\right)[28,33]$. 

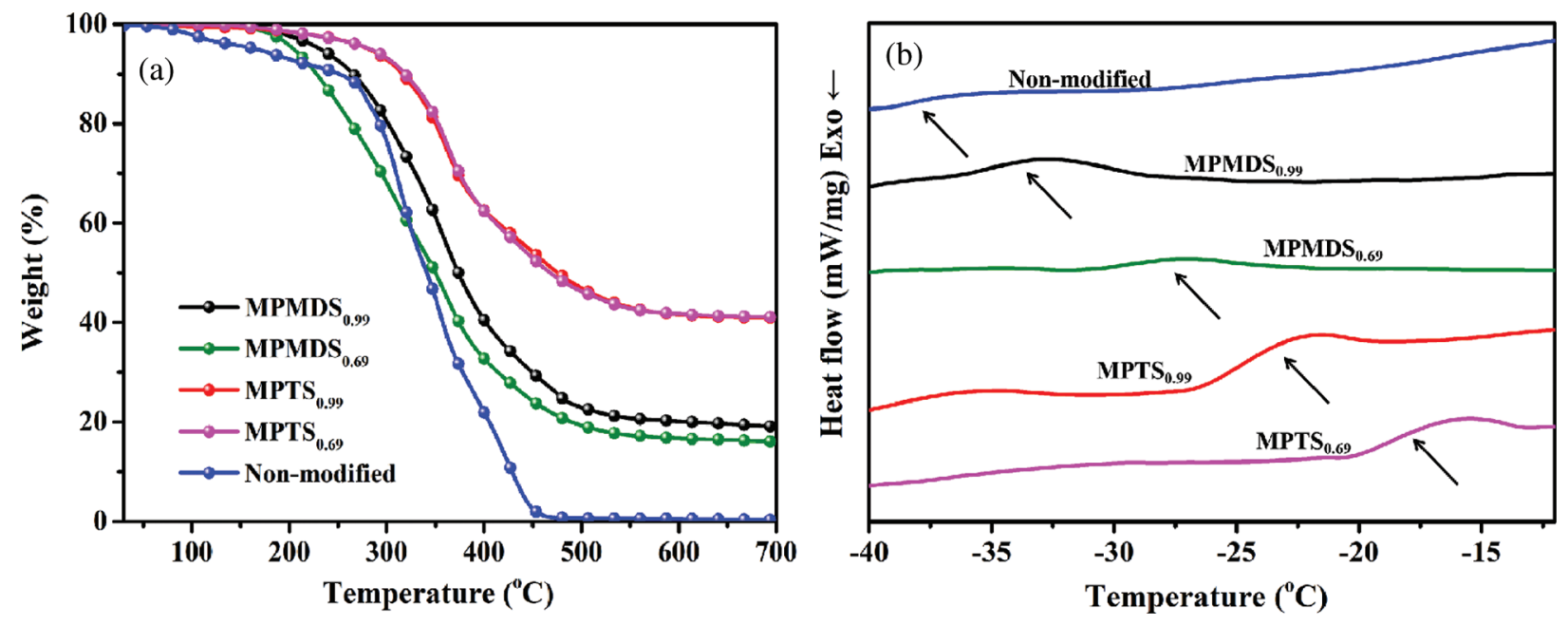

Figure 8: (a) TGA curves of the WPU films modified with different methoxysilanes (b) DSC curves of the WPU films modified with different methoxysilanes

Table 5: TGA data of the WPU films modified with different methoxysilanes

\begin{tabular}{lllll}
\hline Sample & $T_{5}\left({ }^{\circ} \mathrm{C}\right)$ & $T_{10}\left({ }^{\circ} \mathrm{C}\right)$ & $T_{50}\left({ }^{\circ} \mathrm{C}\right)$ & $T_{\max }\left({ }^{\circ} \mathrm{C}\right)$ \\
\hline MPTS $_{0.99}$ & 281 & 316 & 475 & 362 \\
MPTS $_{0.69}$ & 282 & 318 & 467 & 363 \\
MPMDS $_{0.99}$ & 218 & 251 & 360 & 355 \\
MPMDS $_{0.69}$ & 204 & 227 & 350 & 360 \\
Non-modified & 165 & 251 & 341 & 313 \\
\hline
\end{tabular}

Glass transition temperature $\left(T_{\mathrm{g}}\right)$ is the temperature of an amorphous polymer (including the amorphous portion of the crystalline polymer) with transition from a glassy state to a high elastic state or from the latter to the former [34,35]. DSC measurements have been carried out to determine the effect of the MPTS or MPMDS modification on the $T_{\mathrm{g}}$ of the WPU. Obviously, only one $T_{\mathrm{g}}$ and no melting or crystallization transition are observed in all DSC curves (see Fig. 8b), indicating the amorphous nature of the modified films. On the one hand, films modified with MPMDS have lower $T_{\mathrm{g}}\left(-35^{\circ} \mathrm{C} \sim 28^{\circ} \mathrm{C}\right)$ than films modified with MPTS $\left(-24^{\circ} \mathrm{C} \sim-17^{\circ} \mathrm{C}\right)$. On the other hand, All the modified WPU films have higher $T_{\mathrm{g}}$ than nonmodified film $\left(-38^{\circ} \mathrm{C}\right)$. This is because the high content of methoxy groups leads to high hydrolysis capacity and thus increases the cross-linked density.

\section{Conclusions}

In this study, a series of castor oil based waterborne anionic polyurethane were successfully prepared followed by silane modification through thiol-ene photo click reaction. It is found that the particle size and zeta potential of the WPU dispersions both decreased with the rise of emulsifier molar ratios. All the WPU films exhibit excellent water resistance, solvent resistance in acid and salt solution. The modified WPU films showed highly water resistance which $72 \mathrm{~h}$ water absorption could be reduced to $1.94 \%$ and contact angle was up to $99.34^{\circ}$. Besides, the contact angle and thermal stability of the films modified 
with MPMDS are lower than those modified with MPTS. In general, this work provides a new way of siloxane modification to form a cross-linked network for high water resistance performance of WPU.

Funding Statement: This work was sponsored by the National Key Research and Development Program of China (2019YFD1101202, 2019YFD1101203), Guangdong Province Science \& Technology Program (2018B030306016), Guangdong Provincial Innovation Team for General Key Technologies in Modern Agricultural Industry (2019KJ133), Key Projects of Basic Research and Applied Basic Research of the Higher Education Institutions of Guangdong Province (2018KZDXM014), Guangzhou Municipal Key Laboratory of Woody Biomass Functional New Materials (201905010005).

Conflicts of Interest: The authors declare that they have no conflicts of interest to report regarding the present study.

\section{References}

1. Lu, Y., Larock, R. C. (2008). Soybean-oil-based waterborne polyurethane dispersions: Effects of polyol functionality and hard segment content on properties. Biomacromolecules, 9(11), 3332-3340. DOI 10.1021/bm801030g.

2. Rahman, M. M., Kim, E. Y., Yun Kwon, J., Yoo, H. J., Kim, H. D. (2008). Cross-linking reaction of waterborne polyurethane adhesives containing different amount of ionic groups with hexamethoxymethyl melamine. International Journal of Adhesion and Adhesives, 28(1-2), 47-54. DOI 10.1016/j.jjadhadh.2007.03.004.

3. Zhang, C., Garrison, T. F., Madbouly, S. A., Kessler, M. R. (2017). Recent advances in vegetable oil-based polymers and their composites. Progress in Polymer Science, 71, 91-143. DOI 10.1016/j. progpolymsci.2016.12.009.

4. Dhoke, S. K., Sinha, T. J. M., Dutta, P., Khanna, A. S. (2008). Formulation and performance study of low molecular weight, alkyd-based waterborne anticorrosive coating on mild steel. Progress in Organic Coatings, 62(2), 183-192. DOI 10.1016/j.porgcoat.2007.10.008.

5. Liang, H., Wang, S., He, H., Wang, M., Liu, L. et al. (2018). Aqueous anionic polyurethane dispersions from castor oil. Industrial Crops and Products, 122, 182-189. DOI 10.1016/j.indcrop.2018.05.079.

6. Mo, Q., Li, W., Yang, H., Gu, F., Chen, Q. et al. (2019). Water resistance and corrosion protection properties of waterborne polyurethane coating enhanced by montmorillonite modified with $\mathrm{Ce}^{3+}$. Progress in Organic Coatings, 136, 105213. DOI 10.1016/j.porgcoat.2019.105213.

7. Yu, F., Cao, L., Meng, Z., Lin, N., Liu, X. Y. (2016). Crosslinked waterborne polyurethane with high waterproof performance. Polymer Chemistry, 7(23), 3913-3922. DOI 10.1039/c6py00350h.

8. Zhang, W., Zhang, Y., Liang, H., Liang, D., Cao, H. et al. (2019). High bio-content castor oil based waterborne polyurethane/sodium lignosulfonate composites for environmental friendly UV absorption application. Industrial Crops and Products, 142, 111836. DOI 10.1016/j.indcrop.2019.111836.

9. Zhang, W., Deng, H., Xia, L., Shen, L., Zhang, C. et al. (2021). Semi-interpenetrating polymer networks prepared from castor oil-based waterborne polyurethanes and carboxymethyl chitosan. Carbohydrate Polymers, 256(1), 117507. DOI 10.1016/j.carbpol.2020.117507.

10. Gurunathan, T., Mohanty, S., Nayak, S. K. (2015). Effect of reactive organoclay on physicochemical properties of vegetable oil-based waterborne polyurethane nanocomposites. RSC Advances, 5(15), 11524-11533. DOI 10.1039/ c4ra14601h.

11. Dai, Z., Jiang, P., Lou, W., Zhang, P., Bao, Y. et al. (2020). Preparation of degradable vegetable oil-based waterborne polyurethane with tunable mechanical and thermal properties. European Polymer Journal, 139(12), 109994. DOI 10.1016/j.eurpolymj.2020.109994.

12. Liang, H., Lu, Q., Liu, M., Ou, R., Wang, Q. et al. (2020). UV absorption, anticorrosion, and long-term antibacterial performance of vegetable oil based cationic waterborne polyurethanes enabled by amino acids. Chemical Engineering Journal, 421. DOI 10.1016/j.cej.2020.127774. 
13. Liu, L., Deng, H., Zhang, W., Madbouly, S. A., He, Z. et al. (2021). Novel internal emulsifiers for high biocontent sustainable pressure sensitive adhesives. ACS Sustainable Chemistry \& Engineering, 9(1), 147-157. DOI 10.1021/ acssuschemeng.0c05936.

14. Wang, X., Liang, H., Jiang, J., Wang, Q., Luo, Y. et al. (2020). A cysteine derivative-enabled ultrafast thiol-ene reaction for scalable synthesis of a fully bio-based internal emulsifier for high-toughness waterborne polyurethanes. Green Chemistry, 22(17), 5722-5729. DOI 10.1039/D0GC02213F.

15. Liu, K., Miao, S., Su, Z., Sun, L., Ma, G. et al. (2016). Castor oil-based waterborne polyurethanes with tunable properties and excellent biocompatibility. European Journal of Lipid Science and Technology, 118(10), 15121520. DOI 10.1002/ejlt.201500595.

16. Zhang, Y., Zhang, W., Wang, X., Dong, Q., Zeng, X. et al. (2020). Waterborne polyurethanes from castor oil-based polyols for next generation of environmentally-friendly hair-styling agents. Progress in Organic Coatings, 142, 105588. DOI 10.1016/j.porgcoat.2020.105588.

17. Zhang, C., Liang, H., Liang, D., Lin, Z., Chen, Q. et al. (2021). Renewable castor-oil-based waterborne polyurethane networks: Simultaneously showing high strength, self-healing, processability and tunable multishape memory. Angewandte Chemie International Edition in English, 60(8), 4289-4299. DOI 10.1002/ anie.202014299.

18. Zhang, Y., Zhang, W., Deng, H., Zhang, W., Kang, J. et al. (2020). Enhanced mechanical properties and functional performances of cationic waterborne polyurethanes enabled by different natural phenolic acids. ACS Sustainable Chemistry \& Engineering, 8(47), 17447-17457. DOI 10.1021/acssuschemeng.0c05883.

19. Wei, D., Liao, B., Yong, Q., Wang, H., Li, T. et al. (2019). Castor oil-based waterborne hyperbranched polyurethane acrylate emulsion for UV-curable coatings with excellent chemical resistance and high hardness. Journal of Coatings Technology and Research, 16(2), 415-428. DOI 10.1007/s11998-018-0120-1.

20. Fang, Z. H., Duan, H. Y., Zhang, Z. H., Wang, J., Li, D. Q. et al. (2011). Novel heat-resistance UV curable waterborne polyurethane coatings modified by melamine. Applied Surface Science, 257(11), 4765-4768. DOI 10.1016/j.apsusc.2010.10.082.

21. Li, J., Cui, J., Yang, J., Li, Y., Qiu, H. et al. (2016). Reinforcement of graphene and its derivatives on the anticorrosive properties of waterborne polyurethane coatings. Composites Science and Technology, 129(2), 3037. DOI 10.1016/j.compscitech.2016.04.017.

22. Chen, R., Chen, J. S., Zhang, C., Kessler, M. R. (2015). Rapid room-temperature polymerization of bio-based multiaziridine-containing compounds. RSC Advances, 5(2), 1557-1563. DOI 10.1039/C4RA10335A.

23. Tejado, A., Antal, M., Liu, X., van de Ven, T. G. M., (2011). Wet cross-linking of cellulose fibers via a bioconjugation reaction. Industrial \& Engineering Chemistry Research, 50(10), 5907-5913. DOI 10.1021/ ie1023589.

24. Rahman, M. M., Kim, H. D., Lee, W. K. (2009). Properties of crosslinked waterborne polyurethane adhesives with modified melamine: Effect of curing time, temperature, and HMMM content. Fibers and Polymers, 10(1), 6-13. DOI 10.1007/s12221-009-0006-z.

25. Zhang, Q., Liu, W., Sun, F. (2020). Synthesis and properties of waterborne UV-curable polydimethylsiloxanebased polyurethane oligomers: UV-cured film with excellent water resistance and thermostability. Journal of Adhesion Science and Technology, 34(20), 2245-2261. DOI 10.1080/01694243.2020.1757191.

26. Kim, H. A., Kim, B. K. (2019). Synthesis and properties of waterborne polyurethane/hydroxyapatite chemical hybrids. Progress in Organic Coatings, 128, 69-74. DOI 10.1016/j.porgcoat.2018.12.009.

27. Wu, G., Zang, H., Zhang, H. (2020). Preparation and performance of UV-curable waterborne polyurethane prepared using dipentaerythritol hexaacrylate/dipropylene glycol diacrylate monomers. Journal of Macromolecular Science-Part A, 57(12), 927-934. DOI 10.1080/10601325.2020.1807364.

28. Dang, X., Li, Y., Yang, M. (2019). Biodegradable waterborne polyurethane grafted with gelatin hydrolysate via solvent-free copolymerization for potential porous scaffold material. Journal of the Mechanical Behavior of Biomedical Materials, 92(10), 79-89. DOI 10.1016/j.jmbbm.2019.01.005.

29. Zheng, G., Lu, M., Rui, X., Shao, B. (2018). Surface and bulk properties of waterborne polyurethane modified with fluorinated siloxane. Journal of Applied Polymer Science, 135(28), 46473. DOI 10.1002/app.46473. 
30. Wu, Y., Du, Z., Wang, H., Cheng, X. (2017). Synthesis of aqueous highly branched silica sol as underlying crosslinker for corrosion protection. Progress in Organic Coatings, 111, 381-388. DOI 10.1016/j. porgcoat.2017.06.023.

31. Fan, W., Jin, Y., Shi, L. (2020). Mechanically robust and tough waterborne polyurethane films based on diselenide bonds and dual H-bonding interactions with fast visible-light-triggered room-temperature self-healability. Polymer Chemistry, 11(34), 5463-5474. DOI 10.1039/D0PY00897D.

32. Xia, Y., Zhang, Z., Kessler, M. R., Brehm-Stecher, B., Larock, R. C. (2012). Antibacterial soybean-oil-based cationic polyurethane coatings prepared from different amino polyols. ChemSusChem, 5(11), 2221-2227. DOI $10.1002 /$ cssc. 201200352.

33. Xu, J., Jiang, Y., Zhang, T., Dai, Y., Yang, D. et al. (2018). Synthesis of UV-curing waterborne polyurethaneacrylate coating and its photopolymerization kinetics using FT-IR and photo-DSC methods. Progress in Organic Coatings, 122, 10-18. DOI 10.1016/j.porgcoat.2018.05.008.

34. Peng, L., Zhou, L., Li, Y., Pan, F., Zhang, S. (2011). Synthesis and properties of waterborne polyurethane/ attapulgite nanocomposites. Composites Science and Technology, 71(10), 1280-1285. DOI 10.1016/j. compscitech.2011.04.012.

35. Liang, H., Li, Y., Huang, S., Huang, K., Zeng, X. et al. (2019). Tailoring the performance of vegetable oil-based waterborne polyurethanes through incorporation of rigid cyclic rings into soft polymer networks. ACS Sustainable Chemistry \& Engineering, 8(2), 914-925. DOI 10.1021/acssuschemeng.9b05477. 